\title{
Mindfulness-based emotional regulation for patients with implantable cardioverter-defibrillators: A randomized pilot study of efficacy, applicability, and safety
}

\author{
Santiago Montero Ruiz ${ }^{1}$, Beatriz Rodríguez Vega ${ }^{2}$, \\ Carmen Bayón Pérez ${ }^{2}$, Rafael Peinado Peinado ${ }^{3}$ (1) \\ ${ }^{1}$ Faculty of Medicine, Universidad Autónoma de Madrid, Spain \\ ${ }^{2}$ Liaison and Psychotherapy Unit, Department of Psychiatry, Hospital Universitario La Paz, \\ Universidad Autónoma de Madrid, Spain \\ ${ }^{3}$ Arrhythmia Unit, Cardiology Department, Hospital Universitario La Paz, \\ Universidad Autónoma de Madrid, Spain
}

\begin{abstract}
Background: The efficacy of mindfulness-based interventions to reduce anxiety or improve quality of life (QoL) in patients with cardiac pathologies is well established. However, there is scarce information on the efficacy, applicability, and safety of these interventions in adult patients with an implantable cardioverter-defibrillator (ICD). In this study, we examined their efficacy on QoL, psychological and biomedical variables, as well as the applicability and safety of a mindfulness-based intervention in patients with an ICD.
\end{abstract}

Methods: Ninety-six patients with an ICD were randomized into two intervention groups and a control group. The interventions involved training in mindfulness-based emotional regulation, either face-to-face or using the "REM Volver a casa" mobile phone application (app).

Results: The sample presented medium-high QoL baseline scores (mean: 68), low anxiety (6.84) and depression (3.89), average mindfulness disposition (128), and cardiological parameters similar to other ICD populations. After the intervention, no significant differences were found in the variables studied between the intervention and control groups. Retention was average (59\%), and there were no adverse effects due to the intervention.

Conclusions: After training in mindfulness-based emotional regulation (face-to-face or via app), no significant differences were found in the QoL or psychological or biomedical variables in patients with an ICD. The intervention proved to be safe, with $59 \%$ retention. (Cardiol J)

Key words: quality of life, implantable cardioverter-defibrillator, emotional regulation, mindfulness, anxiety

\section{Introduction}

Stress, anxiety, and depression are some of the psychological disorders associated with pathologies of the cardiovascular system, affecting cognitive performance and quality of life (QoL) [1-3].

Mindfulness is a complementary intervention in the treatment of problems such as anxiety,

Address for correspondence: Ass. Prof. Rafael Peinado Peinado, Arrhythmia Unit, Cardiology Department, Hospital Universitario La Paz. Paseo de la Castellana, 261, 28049 Madrid, Spain, tel: +34 620228084, e-mail: rpeinado@secardiologia.es

This article is available in open access under Creative Common Attribution-Non-Commercial-No Derivatives 4.0 International (CC BY-NC-ND 4.0) license, allowing to download articles and share them with others as long as they credit the authors and the publisher, but without permission to change them in any way or use them commercially. 
which has demonstrated psychological benefits $[2,4-7]$. It has also been shown to be effective in cardiovascular diseases $[4,8]$, facilitating greater emotional stability [9-12] and improving mood in patients with heart disease $[3,8,13]$.

The implanted cardioverter-defibrillator (ICD) is a first-line treatment in patients who have had poorly tolerated ventricular tachyarrhythmias or who have had resuscitated cardiac arrest. It also plays a fundamental role in the primary prevention of sudden cardiac death in patients with heart disease and risk of developing malignant ventricular arrhythmias. After ICD implantation, many of these patients suffer not only from the fear of arrhythmias and their possible consequences, but also (and especially) from the shocks that the ICD can deliver to treat arrhythmias [14, 15].

In patients with heart disease, Younge et al. [1] obtained positive results in the physiological parameters (exercise capacity and heart rate) assessed after an online mindfulness-based intervention. However, there is scarce information on the influence of this type of intervention in patients with ICDs. Salmoirago-Blotcher et al. [12, 16, 17] conducted mindfulness-based interventions in this type of patient. Their results showed a significant increase in participants' levels of mindfulness but did not have a significant effect on anxiety symptoms. In addition, they did not study the effect on QoL or the biomedical variables of the patients.

The main objective of this pilot study was to analyze the efficacy, applicability, and safety of a mindfulness-based intervention administered face-to-face or through an application (app) on QoL, psychological, and biomedical variables in a sample of patients with an ICD. The secondary objective was to describe the baseline characteristics of QoL, psychological, and biomedical variables in this population.

\section{Methods}

\section{Design and study population}

A randomized controlled clinical trial was conducted with a pre-post design.

The study population consisted of patients with heart disease and an ICD treated in the Arrhythmia Unit of the Cardiology Department of a Spanish university hospital. In total, 340 patients met the inclusion criteria, which were as follows: being over 18 years of age; having an ICD that had been implanted 3 months ago or more; having sufficient knowledge of Spanish; ownership of a mobile phone and the ability to use mobile apps; and sufficient availability and physical condition to attend face-to-face training in emotional regulation.

The exclusion criteria were as follows: being over 75 years of age; being on the waiting list for a heart transplant or other heart-related surgery; being a current mindfulness practitioner or having been one during the last 5 years; or having a severe mental disorder in an acute period.

The sample size was calculated to detect a difference greater than or equal to 10 units in the QoL variable, considering an alpha risk of 0.05 and beta of 0.2 , in a bilateral contrast, as well as a standard deviation of 20 points and a loss rate of $20 \%$. The estimated number of patients was 237 , to be distributed into three groups of 79 patients each.

Patients were recruited by telephone and were randomized using the Excel function [=RANDBETWEEN $(1 ; 3)]$. The participants were randomly distributed into three groups: two for the intervention (face-to-face group and app group) and a non-intervention control group.

\section{Measuring instruments}

- SF-36 (Short Form): Health-related QoL survey [18];

- HADS (Hospital Anxiety and Depression Scale): Scale designed to assess the presence of anxiety or depression in the medical patient [19];

- STAXI (State-Trait Anger Expression Inventory): Questionnaire that assesses the expression of anger, both as a personality trait and with respect to the state at the time of measurement [20];

- FFMQ (Five Facet Mindfulness Questionnaire): This questionnaire explores five factors of mindfulness disposition [21].

\section{Variables}

The main outcome variable was QoL, assessed by the SF-36 questionnaire. We assessed the degree of applicability based on recruitment and retention figures, as well as the safety of both interventions in terms of adverse effects reported by participants. In each session, we asked about the possible occurrence of any psychological discomfort or the existence and degree of negative adverse effects. Any discomfort, harm, or increase in negative psychological symptoms (anxiety, depression, anger) arising directly from the intervention was considered a negative adverse effect [22, 23].

The secondary variables considered were anxiety, depression, anger, mindfulness disposition, and the following biomedical variables: type 
of baseline heart disease of the patient, functional class (FC), left ventricular ejection fraction (LVEF), indication for ICD implantation, time since implantation, history of ICD therapies, and pharmacological treatment and its changes during the intervention period. The information provided by the ICD itself was also analyzed: therapies both appropriate and inappropriate, non-sustained ventricular tachycardias, mean heart rate, and daily activity of the patient. The data provided by the ICD were compared by considering the 2 months before and the 2 months after the intervention. Ninety-two percent of the patients had remote ICD monitoring.

The independent variable was participation in a mindfulness-based emotional regulation program, either in person or through an app.

The study protocol was approved by the hospital's Clinical Research Ethics Committee.

Trial registration: ClinicalTrials.gov, identifier NCT04235881.

\section{Interventions}

Face-to-face group. A face-to-face 8-week group training program was conducted, with a maximum of 25 participants, based on the mindfulness-based stress reduction program designed by Kabat-Zinn. It included all the elements of the original training program, except that the sessions were 2 hours long and the 7-hour silent practice day was not carried out. The program was taught by 2 accredited mindfulness-based stress reduction teachers.

App group. The intervention was carried out using the "REM Volver a casa" ("REM coming back home") mobile phone application. This application was developed to deliver a user-led 8-week mindfulness-based emotional regulation training program. It was designed by teachers accredited in standardized mindfulness program.

Control group. During the face-to-face and app training period, no intervention was carried out with the participants assigned to the control group. Once the post-intervention data collection was completed, the patients in the control group were offered the mindfulness-based emotional regulation program using the "REM coming back home" app.

\section{Statistical analysis}

In the descriptive analysis, the absolute frequency (n) and relative frequency (\%) were calculated for qualitative variables. For quantitative variables, the normality of the distributions of the variables was tested using the Kolmogorov-Smirnov goodness-of-fit test, expressed as median and interquartile range (IQR). For the analysis of group independence, the Kruskal-Wallis $\mathrm{H}$ test was used for quantitative variables, and the $\chi^{2}$ test (chi-square with Fisher's exact test) was used for categorical variables.

The efficacy of the intervention was analyzed by comparisons between the three study groups. An additional analysis was also chosen, pooling the two intervention groups and comparing the overall group with the control group. The tool used was the Mann-Whitney U test.

For the analysis of drop-out and retention of the study participants, we used the $\chi^{2}$ test (chi-square) for categorical variables and the Mann-Whitney U test for quantitative variables.

For all tests, the p-values for all results were bilateral; a value of $<0.05$ indicated statistical significance.

Statistical analyses were performed using SPSS, version 20 (IBM Corp., United States).

\section{Results}

A total of 251 patients were randomized, of whom 96 started the study and 57 completed it (Fig. 1).

\section{Baseline characteristics}

The sample, $74 \%$ male, had a median age of 60 years (49-67). The median age was higher in the face-to-face group (65 [53-71]) than in the app group (59 [47-64; $\mathrm{p}=0.013]$ ) or the control group (56 [46-67; $\mathrm{p}=0.052]$ ), and it was higher in men (61 [51-68]) than in women (53 [46-61; $\mathrm{p}=044])$. Almost half $(49 \%)$ of the participants in the app group had a university education, compared to just over a third (36\%) in the face-to-face group.

Regarding baseline heart disease, half ( $52 \%)$ of the participants had ischemic heart disease with previous myocardial infarction; slightly fewer than half (41\%) had non-ischemic cardiomyopathy, mainly dilated, and a minority (7\%) had primary electrical disease. Median LVEF was 31\% (25-50). $45.8 \%$ were in FC II and $14.6 \%$ were in FC III. Fewer patients in FC III were found in the face-to-face group (2.8\%) than in the app (17.1\%) and control (31.6\%) groups. In slightly more than half (56\%) of participants, the implant was performed as primary prevention; in $44 \%$ it was performed as secondary prevention. The mean time from implantation to completion of the study was $2.9 \pm$ \pm 2.14 years. 


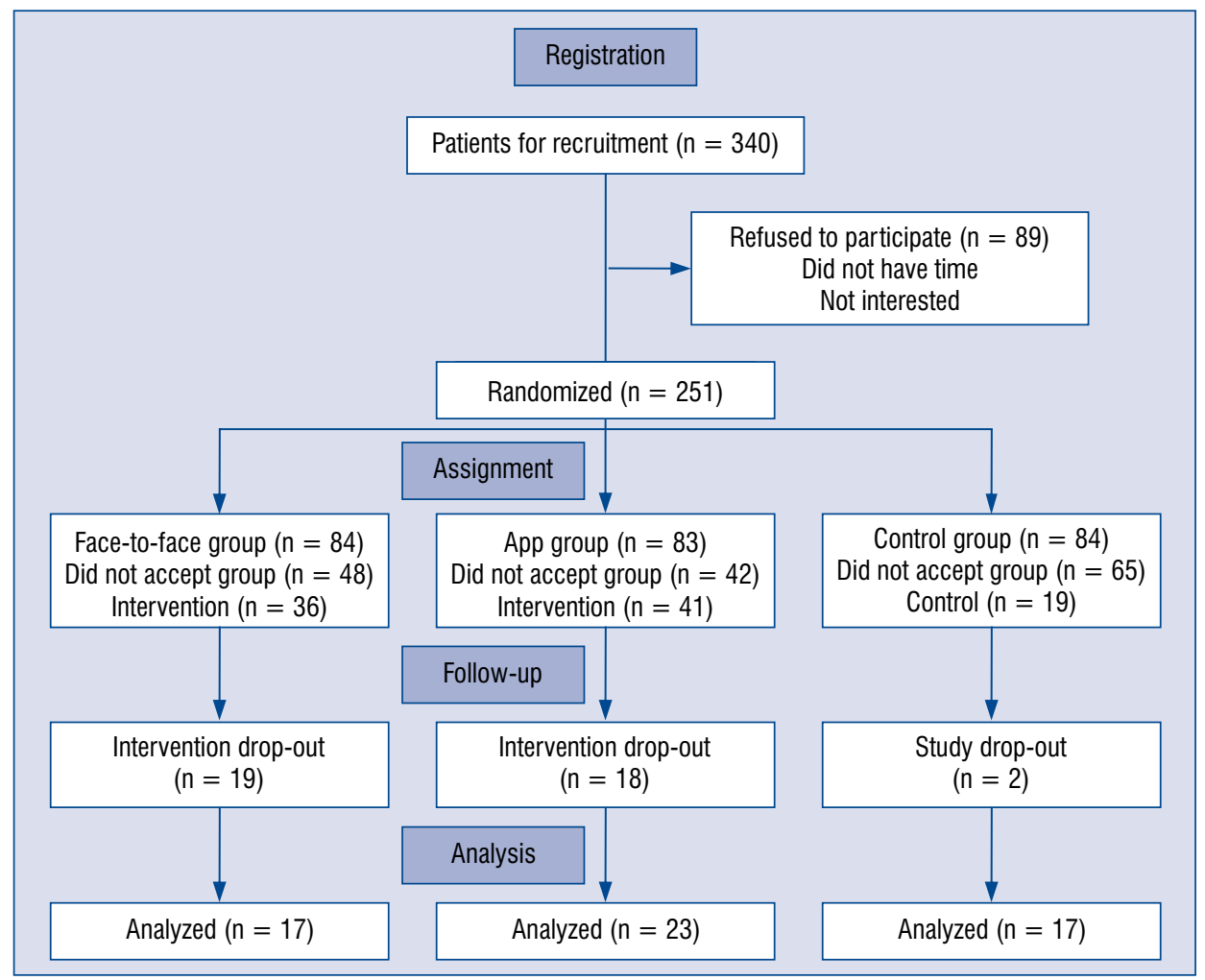

Figure 1. Study flowchart.

Twenty-three percent of the study participants had shocks in the period between implantation and the completion of the study (mean time of 2 years).

Quality of life scores were medium-high (73 [65-90]), low anxiety (6 [4-9]) and depression (3 [1-5.5]), and average in mindfulness disposition (128 [118-139]).

Except for the age variable and $\mathrm{FC}$, there were no statistically significant differences between the groups (Table 1).

\section{Results of the intervention}

No significant differences were found between the intervention and control groups for QoL, anxiety, anger, or depression scores or biomedical variables (appropriate or inappropriate therapies, non-sustained ventricular tachycardias, mean heart rate, or mean daily activity). However, for the general health dimension of the QoL variable, the difference corresponding to the contrast between the face-to-face group and the control group was significant (Table 2).

In the comparison between the two intervention groups grouped together (face-to-face and app) and the control group, statistical significance was not reached in any of the variables (Table 3). The comparison test between secondary studies $(n=10)$ vs. university studies $(n=24)$ only reported statistically significant differences $(p=0.040)$ in the mental health dimension of the QoL variable. Due to the low number of patients, no comparisons were made with other educational levels.

\section{Applicability}

Recruitment reached almost $74 \%$ of potential participants. After randomization and allocation of participants to the study groups, the drop-out rate was $62 \%$. Subsequently, during the intervention, the drop-out rate was $40 \%$. Retention was average in the face-to-face (47\%) and app (56\%) groups, with no significant difference between them ( $\mathrm{p}=0.497)$. In the control group, retention was high (89\%), with a significant difference compared to the face-to-face $(\mathrm{p}=0.003)$ and app $(\mathrm{p}=0.017)$ groups. Female retention (80\%) was higher than male retention $(52 \% ; \mathrm{p}=0.018)$. Apart from group type and sex, no other variables related to drop-out or retention in the study were identified.

\section{Safety}

During the sessions, participants in the face-to-face group reported no negative adverse effects, such as anxiety, stress, low mood, or other psychological distress. Nor did the app group participants 
Table 1. Study variables.

\begin{tabular}{|c|c|c|c|c|c|}
\hline & \multicolumn{3}{|c|}{ Participant characteristics: baseline data $(n=96)$} & \multirow{2}{*}{$\begin{array}{c}\text { Test H } \\
\left(0, \chi^{2}\right)\end{array}$} & \multirow{2}{*}{$\begin{array}{c}P \\
\text { value }\end{array}$} \\
\hline & $\begin{array}{c}\text { Face-to-face } \\
\text { group } \\
(n=36)\end{array}$ & $\begin{array}{c}\text { Application } \\
\text { group } \\
(n=41)\end{array}$ & $\begin{array}{l}\text { Control } \\
\text { group } \\
(n=19)\end{array}$ & & \\
\hline \multicolumn{6}{|l|}{ Sociodemographic } \\
\hline Age [years] & $65(53-71)$ & $59(47-64)$ & $56(46-67)$ & $(2)=7.170$ & $0.028 *$ \\
\hline Sex: & & & & $\chi^{2}=0.464$ & 0.869 \\
\hline Female & $9(25.0 \%)$ & $12(29.3 \%)$ & $4(21.1 \%)$ & & \\
\hline Male & $27(75.0 \%)$ & $29(70.7 \%)$ & $15(78.9 \%)$ & & \\
\hline Educational level: & & & & $\chi^{2}=3.672$ & $>741$ \\
\hline None & $4(11.1 \%)$ & $4(9.7 \%)$ & $2(10.5 \%)$ & & \\
\hline Primary & $8(22.2 \%)$ & $7(17.1 \%)$ & $4(21.1 \%)$ & & \\
\hline Secondary & $11(30.6 \%)$ & $10(24.4 \%)$ & $8(42.1 \%)$ & & \\
\hline University & $13(36.1 \%)$ & $20(48.8 \%)$ & $5(26.3 \%)$ & & \\
\hline \multicolumn{6}{|c|}{ Cardiology/implantable cardioverter-defibrillator } \\
\hline Functional class: & & & & $\chi^{2}=10.118$ & $0.034^{*}$ \\
\hline 1 & $15(41.7 \%)$ & $15(36.6 \%)$ & $8(42.1 \%)$ & & \\
\hline II & $20(55.5 \%)$ & $19(46.3 \%)$ & $5(26.3 \%)$ & & \\
\hline III & $1(2.8 \%)$ & $7(17.1 \%)$ & $6(31.6 \%)$ & & \\
\hline IV & $0(0.0 \%)$ & $0(0.0 \%)$ & $0(0.0 \%)$ & & \\
\hline Prevention: & & & & $\chi^{2}=4.285$ & $>127$ \\
\hline Primary & $25(69.4 \%)$ & $19(46.3 \%)$ & $10(52.6 \%)$ & & \\
\hline Secondary & $11(30.6 \%)$ & $22(53.7 \%)$ & $9(47.4 \%)$ & & \\
\hline Pathology: & & & & $\chi^{2}=1.046$ & 0.927 \\
\hline No heart disease & $2(5.5 \%)$ & $4(9.8 \%)$ & $1(5.4 \%)$ & & \\
\hline Ischemic & $20(55.6 \%)$ & $21(51.2 \%)$ & $9(47.3 \%)$ & & \\
\hline Non-ischemic & $14(38.9 \%)$ & $16(39.0 \%)$ & $9(47.3 \%)$ & & \\
\hline Shocks: & & & & $\chi^{2}=1.728$ & 0.440 \\
\hline No & $30(83.3 \%)$ & $31(75.6 \%)$ & $13(68.4 \%)$ & & \\
\hline Yes & $6(16.7 \%)$ & $10(24.4 \%)$ & $6(31.6 \%)$ & & \\
\hline Implant years & $3.0(2.0-3.4)$ & $3.0(2.5-3.9)$ & $3.0(2.4-3.6)$ & 0.368 & \\
\hline LVEF & $33(28-49)$ & $34(30-50)$ & $28(25-50)$ & $(2)=2.834$ & 0.242 \\
\hline Mean heart rate & $65.5(62.5-70)$ & $65.0(65-70)$ & $65.0(60-70)$ & $(2)=0.495$ & 0.781 \\
\hline NSVT & $0(0-1)$ & $0(0-1)$ & $0(0-0)$ & $(2)=0.677$ & 0.713 \\
\hline Daily activity [h] & $3(2-3.4)$ & $3(2.5-3.9)$ & $3(2.4-3.6)$ & $(2)=0.641$ & 0.726 \\
\hline \multicolumn{6}{|l|}{ Quality of life } \\
\hline Physical functioning & $70.0(60.0-85.0)$ & $85.0(65-95)$ & $80.0(60-95)$ & $(2)=3.427$ & 0.180 \\
\hline Role physical & $100(62.5-100)$ & $100(50-100)$ & $100(25-100)$ & $(2)=0.209$ & 0.901 \\
\hline Bodily pain & $72.0(60.5-84.0)$ & $72(62-90)$ & $74(51-84)$ & $(2)=0.513$ & 0.774 \\
\hline General health & $48.5(31.0-59.5)$ & $52.0(37-72)$ & $52.0(40-77)$ & $(2)=1.703$ & 0.427 \\
\hline Vitality & $52.5(47.5-75.0)$ & $65.0(55-80)$ & $55.0(30-75)$ & $(2)=3.536$ & 0.171 \\
\hline Social functioning & $87.5(62.5-100)$ & $87.5(62.5-100)$ & $75.0(50-100)$ & $(2)=1.383$ & 0.501 \\
\hline Role emotional & $100.0(0.0-100)$ & $100(33.3-100)$ & $100(0-100)$ & $(2)=1.528$ & 0.466 \\
\hline Mental health & $74.0(58.0-84.0)$ & $76.0(60-88)$ & $64(48-84)$ & $(2)=1.441$ & 0.487 \\
\hline Physical component summary & $75.65(57.3-87)$ & $82.3(66.7-89)$ & 78 (45.3-93) & $(2)=0.738$ & 0.691 \\
\hline Mental component summary & $80.2(47.6-93.3)$ & $86.5(62.6-96)$ & $71.1(39.5-94)$ & $(2)=1.516$ & 0.469 \\
\hline Overall & $51.0(45.5-65.5)$ & $58.5(46.0-76)$ & $51.0(35-73.5)$ & $(2)=2.345$ & 0.310 \\
\hline
\end{tabular}


Table 1 (cont.). Study variables.

\begin{tabular}{|c|c|c|c|c|c|}
\hline & \multicolumn{3}{|c|}{ Participant characteristics: baseline data $(n=96)$} & \multirow{2}{*}{$\begin{array}{c}\text { Test H } \\
\left(0, \chi^{2}\right)\end{array}$} & \multirow{2}{*}{$\begin{array}{c}P \\
\text { value }\end{array}$} \\
\hline & $\begin{array}{c}\text { Face-to-face } \\
\text { group } \\
(n=36)\end{array}$ & $\begin{array}{c}\text { Application } \\
\text { group } \\
(n=41)\end{array}$ & $\begin{array}{l}\text { Control } \\
\text { group } \\
(n=19)\end{array}$ & & \\
\hline \multicolumn{6}{|l|}{ Psychological variables } \\
\hline Anxiety & $6.0(4.0-10.0)$ & $6.0(3.0-8.0)$ & $7.0(5.0-10.0)$ & $(2)=1.788$ & 0.409 \\
\hline Depression & $3.5(2.0-5.0)$ & $2.0(1.0-5.0)$ & $3.0(1.0-6.0)$ & $(2)=0.837$ & 0.658 \\
\hline Anger (state) & $15.0(15-19)$ & $15.0(15-16)$ & $15.0(15-17)$ & $(2)=1.068$ & 0.586 \\
\hline Anger (trait) & $18.0(14-22)$ & $17.0(15-21)$ & $19.0(15-21)$ & $(2)=0.378$ & 0.828 \\
\hline Anger Expression Index & $29.0(21-36)$ & $28.0(19-33)$ & $25.0(19-35)$ & $(2)=0.551$ & 0.759 \\
\hline Mindfulness total & $128(118-140)$ & $127(119-136)$ & $124(109-141)$ & $(2)=0.760$ & 0.684 \\
\hline
\end{tabular}

Data are shown as median (interquartile range) or number (\%). Test: Kruskal-Wallis $\mathrm{H}$, unless specified. $\chi^{2}$ (chi-square); ${ }^{*} \mathrm{P}$ value for alpha $=0.05$; LVEF — left ventricular ejection fraction; NSVT — non-sustained ventricular tachycardia

indicate psychological distress in their individual practices.

\section{Discussion}

To our knowledge, this study is the first randomized clinical trial to study the applicability and efficacy of 2 mindfulness-based interventions: face-to-face and app-based, in adult patients fitted with an ICD, to improve QoL, psychological, and biological variables.

As with other studies [24, 25], the baseline characteristics of our sample showed medium-high values in most of the scales of the QoL variable. The cardiological biomedical characteristics of the population in this study were also similar to those found in other ICD patient populations, such as those of the Spanish Society of Cardiology's ICD Registry [26]. These data, together with the absence of symptoms of anxiety, depression, and anger, suggest that having an ICD, not recently implanted, does not entail lower QoL. In addition, the data would justify the absence of significant changes in the scores obtained after the intervention [27].

Numerous studies have shown that ICD shocks are the main determinant of poorer QoL, increased anxiety, and psychological disturbances [28-30]; in our study, only $23 \%$ of the included patients had experienced a previous ICD shock. In addition, some studies have shown that in the early post-ICD implantation period, there is a greater deterioration in QoL and emotional impairment [31]. In our sample, the proportion of patients who had been implanted less than a year ago was small (28\%).
The small sample size probably explains why some trends towards favorable changes in the intervention groups compared to the control group did not reach statistical significance, except for the improvement in the general health dimension of the QoL variable in the face-to-face group compared to the control group.

Educational level does not seem to affect the results of mindfulness training. However, a higher educational level (university) could have a favorable effect on mental health, an effect that was found in the comparison between the levels of secondary and university studies.

In patients with cardiovascular disease, several studies, reviews, and meta-analyses have shown that this type of intervention has a moderate effect on outcomes relating to psychological variables and unclear effects on biological variables [32]. A recent systematic review and meta-analysis [33] showed an improvement in psychological variables as well as in systolic, although not diastolic, blood pressure. However, the QoL of patients was not analyzed in this review and patients with ICDs were not included. On the other hand, the effect obtained by Salmoirago-Blotcher et al. [16] on anxiety symptoms did not reach statistical significance in the overall group; it was only reached if patients who performed all sessions were considered.

Dash et al. [34], in a pilot study on the effect of meditation in 25 heart failure patients with ICDs, demonstrated a reduction in episodes of atrial fibrillation and sustained ventricular tachycardia. However, we used a rough analysis of these episodes, not adjusted for the number of episodes per patient, which is a major methodological limitation. 


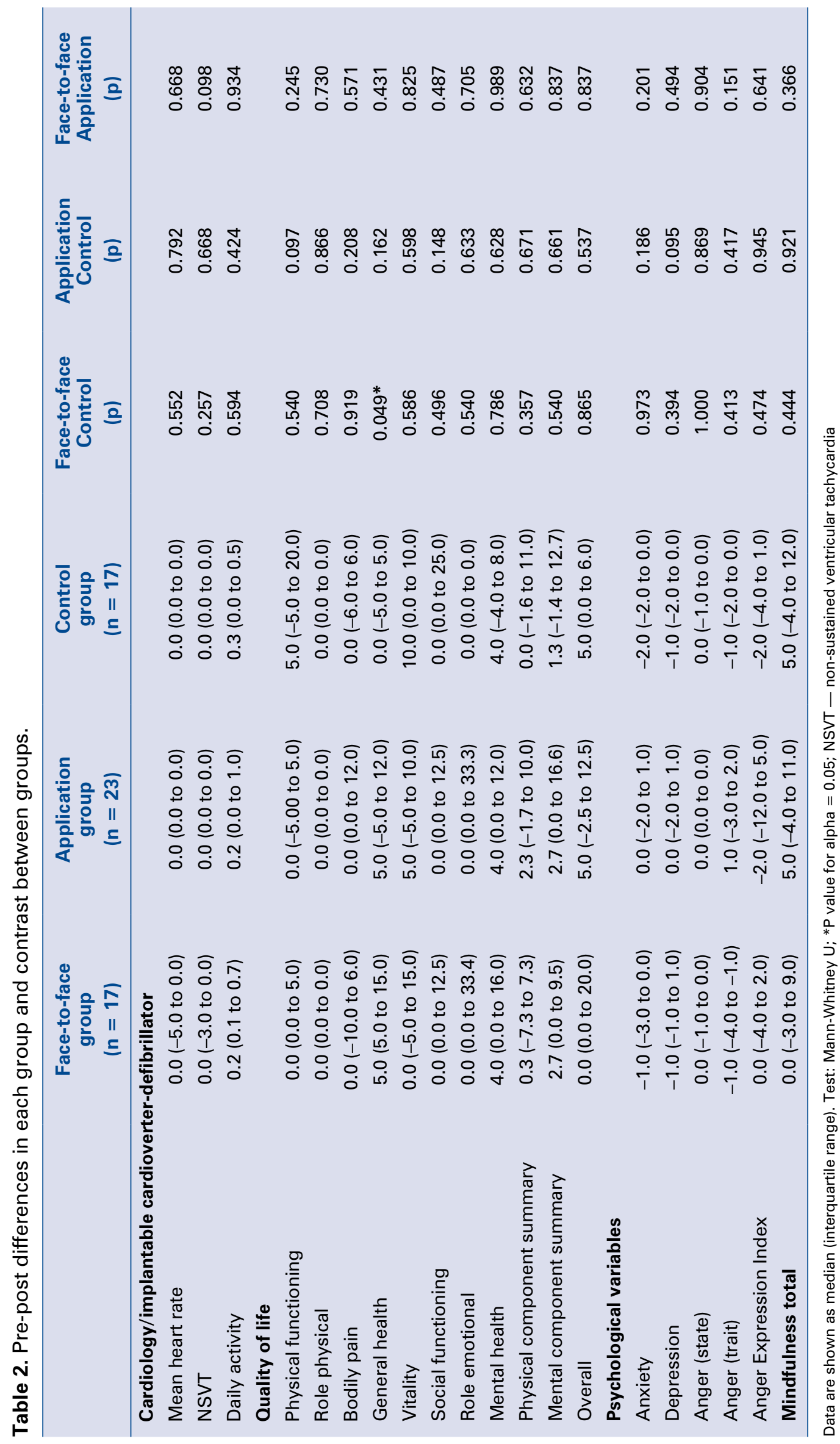


Table 3. Pre-post differences in each group and contrast between joined intervention group and control group.

\begin{tabular}{|c|c|c|c|c|}
\hline & \multicolumn{2}{|c|}{$\begin{array}{l}\text { Participants with intervention } \\
\text { vs. control group }\end{array}$} & \multirow[t]{2}{*}{ Test U } & \multirow[t]{2}{*}{$P$ value } \\
\hline & $\begin{array}{l}\text { Intervention group } \\
\qquad(n=40)\end{array}$ & $\begin{array}{l}\text { Control group } \\
\qquad(\mathrm{n}=17)\end{array}$ & & \\
\hline \multicolumn{5}{|c|}{ Cardiology/implantable cardioverter-defibrillator } \\
\hline Mean heart rate & $0.0(-5.0$ to 0.0$)$ & $0.00(0.00$ to 0.00$)$ & 334.0 & 0.901 \\
\hline NSVT & $0.0(-1.0$ to 0.0$)$ & $0.00(0.00$ to 0.00$)$ & 322.5 & 0.716 \\
\hline Daily activity & $0.2(0.0$ to 0.9$)$ & $0.30(0.00$ to 0.50$)$ & 297.0 & 0.451 \\
\hline \multicolumn{5}{|l|}{ Quality of life } \\
\hline Physical functioning & $0.0(-5.0$ to 5.0$)$ & $5.0(-5.0$ to 20.0$)$ & 262.0 & 0.166 \\
\hline Role physical & $0.0(0.0$ to 0.0$)$ & $0.0(0.0$ to 0.0$)$ & 323.5 & 0.727 \\
\hline Bodily pain & $0.0(-3.0$ to 11.0$)$ & $0.0(-6.0$ to 6.0$)$ & 292.5 & 0.395 \\
\hline General health & $5.0(0.0$ to 13.5$)$ & $0.0(-5.0$ to 5.0$)$ & 232.5 & 0.058 \\
\hline Vitality & $5.0(-5.0$ to 12.5$)$ & $10.0(0.0$ to 10.0$)$ & 304.5 & 0.532 \\
\hline Social functioning & $0.0(0.0$ to 12.5$)$ & $0.0(0.0$ to 25.0$)$ & 271.0 & 0.197 \\
\hline Role emotional & 0.0 (0.0 to 33.3$)$ & $0.0(0.0$ to 0.0$)$ & 307.0 & 0.492 \\
\hline Mental health & $4.0(0.0$ to 12.0$)$ & $4.0(-4.0$ to 8.0$)$ & 314.0 & 0.647 \\
\hline Physical component summary & $1.0(-3.4$ to 8.7$)$ & $0.0(-1.6$ to 11.0$)$ & 297.5 & 0.458 \\
\hline Mental component summary & 2.7 (0.0 to 15.85 & $1.3(-1.4$ to 12.7 & 305.5 & 0.546 \\
\hline Overall & $2.5(-1.25$ to 13.8$)$ & $5.0(0.0$ to 6.0$)$ & 312.0 & 0.624 \\
\hline \multicolumn{5}{|l|}{ Psychological variables } \\
\hline Anxiety & $-0.5(-2.0$ to 0.0$)$ & $-2.0(-2.0$ to 0.0$)$ & 293.5 & 0.411 \\
\hline Depression & $0.0(-1.5$ to 1.0$)$ & $-1.0(-2.0$ to 0.0$)$ & 255.5 & 0.133 \\
\hline Anger (state) & $0.0(-1.0$ to 0.0$)$ & $0.0(-1.0$ to 0.0$)$ & 334.5 & 0.917 \\
\hline Anger (trait) & $-1.0(-4.0$ to 2.0$)$ & $-1.0(-2.0$ to 0.0$)$ & 335.0 & 0.930 \\
\hline Anger Expression Index & $-1.0(-6.0$ to 5.0$)$ & $-2.0(-4.0$ to 1.0$)$ & 316.0 & 0.675 \\
\hline Mindfulness total & $4.0(-4.0$ to 10.0$)$ & $5.0(-4.0$ to 12.0$)$ & 294.0 & 0.637 \\
\hline
\end{tabular}

Data are shown as median (interquartile range). Test: Mann-Whitney U; NSVT — non-sustained ventricular tachycardia

Regarding the lack of effect of the intervention on biomedical variables, in the study by Toise et al. [15], the number of device therapies was lower in the intervention group than in the control group (2014 study). We believe that the absence of differences in our study is mainly due to a short pre- and post-intervention analysis time window and better scheduling of screening and therapies in ICDs.

Despite the high recruitment rate $(74 \%)$, which is much higher than that reported in other studies (Salmoirago-Blotcher et al. [16] - 13\%; Frizelle et al. [35] - 28\%), the sample was greatly reduced due to the high number of dropouts. The patients explained the dropouts as being due to difficulties in participating or a preference for a group other than the assigned group. The dropouts from the control group (77\%) correspond to patients who did not want to be part of this group or did not want to wait a long time to receive training through the application for mobile terminals. It is noteworthy that only $3 \%$ of the patients with FC III were in the face-to-face group, probably because the greater physical demands of this format led them to refuse to participate. On the other hand, some patients assigned to the mobile phone app group gave technical difficulties with the use of the mobile phone as a reason for dropping out.

The mean retention rate (59\%) was far from that reported by Salmoirago-Blotcher et al. [16] $(93 \%)$ or that of the systematic review conducted by Scott-Sheldon [33] (81\%). The lack of explicit follow-up during the intervention may have been the reason for failure to achieve higher retention.

\section{Clinical implications}

The neutral results of this pilot study, in terms of the effects of the intervention in an unselected population of patients fitted with ICDs, suggest that it would probably be more efficient to use it in patients with higher levels of anxiety, depression, 
or stress and poorer QoL, ideally after the onset of ICD shocks.

We did not obtain results that would lead us to think about a better response to training in patients who have suffered ICD discharges. Only 8 patients with shock underwent mindfulness training. Hence, it was not possible to obtain conclusive results regarding training when comparing 8 patients with shocks vs. 32 without shocks. No statistically significant differences were found in the analysis (U Mann-Whitney).

The low retention rate observed in the study and the current low incidence of shocks in patients with ICDs suggest that future studies in this field should be designed as multi-center studies in order to achieve adequate sample sizes.

Finally, it is demonstrated that the app intervention is feasible and can be used for this type of training.

\section{Limitations of the study}

The main limitation of our study was the low number of patients in each group, which meant we were unable to draw conclusive results. Other studies with a much smaller size, such as the 45 participants in the study by Salmoirago-Blotcher et al. [16], found significant results for the anxiety variable.

The high dropout rate in both intervention branches could be an expression of the fact that patients with ICDs do not find this type of tool useful.

Another limitation was that patients with ICD shocks (the main predictor of QoL impairment and anxiety in these patients) or with recent implantation were under-represented.

Finally, a lack of information on attendance at the sessions, or on the use of the app, means that we cannot provide data on treatment compliance.

\section{Conclusions}

After patients with an ICD completed an 8-week mindfulness training program, either face-to-face or through an app, no significant differences were found in QoL, psychological, or biomedical variables.

The intervention program, both face-to-face and via app, was well accepted by participants, with a retention rate of $59 \%$ and no adverse effects reported.

\section{Acknowledgments}

We would like to thank Mr. Roberto Mediavilla Torres for his help in the methodological review of this original work.

Conflict of interest: None declared

\section{References}

1. Younge JO, Wery MF, Gotink RA, et al. Web-Based mindfulness intervention in heart disease: a randomized controlled trial. PLoS One. 2015; 10(12): e0143843, doi: 10.1371/journal. pone.0143843, indexed in Pubmed: 26641099.

2. Abbott RA, Whear R, Rodgers LR, et al. Effectiveness of mindfulness-based stress reduction and mindfulness based cognitive therapy in vascular disease: A systematic review and metaanalysis of randomised controlled trials. J Psychosom Res. 2014; 76(5): 341-351, doi: 10.1016/j.jpsychores.2014.02.012, indexed in Pubmed: 24745774.

3. Schwartz BG, French WJ, Mayeda GS, et al. Emotional stressors trigger cardiovascular events. Int J Clin Pract. 2012; 66(7): 631-639, doi: 10.1111/j.1742-1241.2012.02920.x, indexed in Pubmed: 22698415.

4. Grossman P, Niemann L, Schmidt S, et al. Mindfulness-based stress reduction and health benefits. A meta-analysis. J Psychosom Res. 2004; 57(1): 35-43, doi: 10.1016/S0022-3999(03)00573-7, indexed in Pubmed: 15256293.

5. Tacón AM, McComb J, Caldera Y, et al. Mindfulness meditation, anxiety reduction, and heart disease: a pilot study. Fam Community Health. 2003; 26(1): 25-33, doi: 10.1097/00003727200301000-00004, indexed in Pubmed: 12802125.

6. Loucks EB, Britton WB, Howe CJ, et al. Positive associations of dispositional mindfulness with cardiovascular health: the new england family study. Int J Behav Med. 2015; 22(4): 540-550, doi: 10.1007/s12529-014-9448-9, indexed in Pubmed: 25339282.

7. Loucks EB, Schuman-Olivier Z, Britton WB, et al. Mindfulness and cardiovascular disease risk: state of the evidence, plausible mechanisms, and theoretical framework. Curr Cardiol Rep. 2015; 17(12): 112, doi: 10.1007/s11886-015-0668-7, indexed in Pubmed: 26482755.

8. Olex S, Newberg A, Figueredo VM. Meditation: should a cardiologist care? Int J Cardiol. 2013; 168(3): 1805-1810, doi: 10.1016/j. ijcard.2013.06.086, indexed in Pubmed: 23890919.

9. Teper R, Segal Z, Inzlicht M. Inside the mindful mind: how mindfulness enhances emotion regulation through improvements in executive control. Curr Dir Psychol Sci. 2013; 22(6): 449-454, doi: 10.1177/0963721413495869.

10. Arch JJ, Craske MG. Mechanisms of mindfulness: emotion regulation following a focused breathing induction. Behav Res Ther. 2006; 44(12): 1849-1858, doi: 10.1016/j.brat.2005.12.007, indexed in Pubmed: 16460668.

11. Parswani MJ, Sharma MP, Iyengar Ss. Mindfulness-based stress reduction program in coronary heart disease: A randomized control trial. Int J Yoga. 2013; 6(2): 111-117, doi: 10.4103/09736131.113405, indexed in Pubmed: 23930029.

12. Salmoirago-Blotcher E, Crawford S, Carmody J, et al. Characteristics of dispositional mindfulness in patients with severe cardiac disease. J Evid Based Complementary Altern Med. 2011; 16(3): 218-225, doi: 10.1177/2156587211405525, indexed in Pubmed: 22116792.

13. Nijjar PS, Puppala VK, Dickinson O, et al. Modulation of the autonomic nervous system assessed through heart rate variability by a mindfulness based stress reduction program. Int J Cardiol. 2014; 177(2): 557-559, doi: 10.1016/j.ijcard.2014.08.116, indexed in Pubmed: 25179555.

14. Manzoni GM, Castelnuovo G, Compare A, et al. Psychological effects of implantable cardioverter defibrillator shocks. A review of study methods. Front Psychol. 2015; 6: 39, doi: 10.3389/ fpsyg.2015.00039, indexed in Pubmed: 25698991. 


\section{Cardiology Journal}

15. Toise SCF, Sears SF, Schoenfeld MH, et al. Psychosocial and cardiac outcomes of yoga for ICD patients: a randomized clinical control trial. Pacing Clin Electrophysiol. 2014; 37(1): 48-62, doi: 10.1111/pace.12252, indexed in Pubmed: 23981048.

16. Salmoirago-Blotcher E, Crawford SL, Carmody J, et al. Phonedelivered mindfulness training for patients with implantable cardioverter defibrillators: results of a pilot randomized controlled trial. Ann Behav Med. 2013; 46(2): 243-250, doi: 10.1007/ s12160-013-9505-7, indexed in Pubmed: 23605175.

17. Salmoirago-Blotcher E, Carmody J, Yeh G, et al. Design and methods for a pilot study of a phone-delivered, mindfulnessbased intervention in patients with implantable cardioverter defibrillators. Evid Based Complement Alternat Med. 2012; 2012: 972106, doi: 10.1155/2012/972106, indexed in Pubmed: 22536294.

18. Alonso J, Prieto L, Antó JM. [The Spanish version of the SF-36 Health Survey (the SF-36 health questionnaire): an instrument for measuring clinical results]. Med Clin (Barc). 1995; 104(20): 771-776, indexed in Pubmed: 7783470.

19. Herrero MJ, Blanch J, Peri JM, et al. A validation study of the hospital anxiety and depression scale (HADS) in a Spanish population. Gen Hosp Psych. 2003; 25(4): 277-283, doi: 10.1016/ s0163-8343(03)00043-4.

20. Spielberger CD, Miguel Tobal JJ. Staxi-2: Inventario de Expresión de Ira Estado-Rasgo. TEA; 2001.

21. Cebolla A, García-Palacios A, Soler J, et al. Psychometric properties of the spanish validation of the five facets of mindfulness questionnaire (FFMQ). Eur J Psychiatry. 2012; 26(2): 118-126, doi: 10.4321/s0213-61632012000200005.

22. Duggan C, Parry G, McMurran M, et al. The recording of adverse events from psychological treatments in clinical trials: evidence from a review of NIHR-funded trials. Trials. 2014; 15: 335, doi: 10.1186/1745-6215-15-335, indexed in Pubmed: 25158932.

23. Dimidjian S, Hollon SD. How would we know if psychotherapy were harmful? Am Psychol. 2010; 65(1): 21-33, doi: 10.1037/ a0017299, indexed in Pubmed: 20063907.

24. Pedersen SS, Carter N, Barr C, et al. Quality of life, depression, and anxiety in patients with a subcutaneous versus transvenous defibrillator system. Pacing Clin Electrophysiol. 2019; 42(12): 1541-1551, doi: 10.1111/pace.13828, indexed in Pubmed: 31677279.

25. Johansen JB, Pedersen SS, Spindler H, et al. Symptomatic heart failure is the most important clinical correlate of impaired quality of life, anxiety, and depression in implantable cardioverter-defibrillator patients: a single-centre, cross-sectional study in 610 patients. Europace. 2008; 10(5): 545-551, doi: 10.1093/europace/ eun073, indexed in Pubmed: 18378633.
26. Fernández Lozano I, Osca Asensi J, Alzueta Rodríguez J. Spanish Implantable Cardioverter-defibrillator Registry. 16th Official Report of the Heart Rhythm Association of the Spanish Society of Cardiology (2019). Rev Esp Cardiol (Engl Ed). 2020; 73(12): 1026-1037, doi: 10.1016/j.rec.2020.07.015, indexed in Pubmed: 33039380 .

27. Israelsson J, Thylén I, Strömberg A, et al. Factors associated with health-related quality of life among cardiac arrest survivors treated with an implantable cardioverter-defibrillator. Resuscitation. 2018; 132: 78-84, doi: 10.1016/j.resuscitation.2018.09.002, indexed in Pubmed: 30201535.

28. Schron EB, Exner DV, Yao Q, et al. Quality of life in the antiarrhythmics versus implantable defibrillators trial: impact of therapy and influence of adverse symptoms and defibrillator shocks. Circulation. 2002; 105(5): 589-594, doi: 10.1161/hc0502.103330, indexed in Pubmed: 11827924.

29. Mark D, Anstrom K, Sun J, et al. Quality of Life with Defibrillator Therapy or Amiodarone in Heart Failure. N Engl J Med. 2008; 359(10): 999-1008, doi: 10.1056/nejmoa0706719.

30. Irvine J, Dorian P, Baker B, et al. Quality of life in the Canadian Implantable Defibrillator Study (CIDS). Am Heart J. 2002; 144(2): 282-289, doi: 10.1067/mhj.2002.124049, indexed in Pubmed: 12177646.

31. Pedersen SS, Theuns DA, Jordaens L, et al. Course of anxiety and device-related concerns in implantable cardioverter defibrillator patients the first year post implantation. Europace. 2010; 12(8): 1119-1126, doi: 10.1093/europace/euq154, indexed in Pubmed: 20507853.

32. Richards SH, Anderson L, Jenkinson CE, et al. Psychological interventions for coronary heart disease. Cochrane Database Syst Rev. 2017; 4: CD002902, doi: 10.1002/14651858.CD002902. pub4, indexed in Pubmed: 28452408.

33. Scott-Sheldon LAJ, Gathright EC, Donahue ML, et al. Mindfulness-based interventions for adults with cardiovascular disease: a systematic review and meta-analysis. Ann Behav Med. 2020; 54(1): 67-73, doi: 10.1093/abm/kaz020, indexed in Pubmed: 31167026.

34. Dash A, Malhotra P, Beri N, et al. Meditation for improved clinical outcomes in patients with implantable defibrillators for heart failure- pilot study. J Atr Fibrillation. 2020; 12(6): 2314, doi: 10.4022/jafib.2314, indexed in Pubmed: 33024493.

35. Frizelle DJ, Lewin RJP, Kaye G, et al. Cognitive-behavioural rehabilitation programme for patients with an implanted cardioverter defibrillator: a pilot study. Br J Health Psychol. 2004; 9(Pt 3): 381-392, doi: 10.1348/1359107041557039, indexed in Pubmed: 15296684. 